\title{
USP8 Inhibitor Suppresses HER-2 Positive Gastric Cancer Cell Proliferation and Metastasis via the PI3K/AKT Signaling Pathway
}

This article was published in the following Dove Press journal: OncoTargets and Therapy

Jiangang Sun ${ }^{1} * *$

Dandan Shen ${ }^{2, *}$

Yichao Zheng ${ }^{2}$

Hongmei Ren ${ }^{2}$

Hongmin $\mathrm{Liu}^{2}$

Xiaoping Chen ${ }^{3}$

Yongshun Gao'

'Department of Gastrointestinal Surgery, The First Affiliated Hospital of Zhengzhou University, Zhengzhou, Henan, People's Republic of China; ${ }^{2}$ Key Laboratory of Advanced Pharmaceutical Technology, Zhengzhou University, Zhengzhou, Henan, People's Republic of China; ${ }^{3}$ Department of Hepatic Surgery of Tongji Hospital, Huazhong University of Science and Technology, Wuhan, Hubei, People's Republic of China

*These authors contributed equally to this work

\begin{abstract}
Purpose: Referring to global cancer statistics, the incidence of gastric cancer (GC) was ranked sixth; however, detailed mechanisms underlying its development were not thoroughly investigated. Previous studies have reported that inhibition of ubiquitin-specific peptidase 8 (USP8) induced degradation of several receptor tyrosine kinases, such as epidermal growth factor receptor (EGFR), embryonic stem cells (ESCs), etc. Nevertheless, the regulation of HER-2 by USP8 and the molecular mechanisms controlling their role in the pathogenesis of GC remain unknown.
\end{abstract}

Patients and Methods: A total of 69 patients with histologically confirmed GC were recruited to satisfy the purpose of this study. Initially, tumor samples and GC cell lines were used to detect USP8 and HER-2 levels. Next, MTT and colony formation assays were applied to analyze cell proliferation capability. Cell migration and invasion ability were examined by transwell assays. To examine related mRNA and protein expressions, Western blot assays and quantitative real-time PCR (qRT-PCR) were performed. Immunofluorescence was used to detect the effect of USP8 inhibitor on GC cells. Finally, in vivo experiments were used to examine the effect of USP8 inhibitor.

Results: Patients with USP8 high-expression tumors have shown worse overall survival while opposite results found in patients with low USP8 expressions. Regarding disease prognosis, patients with low expression of USP8 and HER-2 were performed better prognosis, whereas those with overexpression of USP8 and HER-2 shown poor prognosis. USP8 inhibitor significantly inhibited HER-2 positive cell NCI-N87 proliferation and metastasis. In addition, USP8 stabilizes HER-2, preventing it from ubiquitin proteasome-mediated degradation. In vivo studies confirmed that the USP8 inhibitor inhibited HER-2 positive cell NCI-N87 tumor growth. However, it did not affect the HER2-negative cell MGC-803. Careful investigation unraveled that the USP8 inhibitor significantly inhibited NCI-N87 cell proliferation and metastasis via phosphatidylinositol-3-kinases/protein-serine-threonine kinase (PI3K/AKT) pathway.

Conclusion: The USP8 inhibited HER-2 positive GC cell proliferation and migration in vivo and in vitro and probably served as a novel potential therapeutic biomarker for HER-2 positive GC.

Keywords: gastric cancer, proliferation, metastasis, USP8, HER-2, inhibitor, PI3K/AKT pathway

\section{Introduction}

GC occupied the sixth position among all cancer cases with a percentage of 5.7 (18.1 million) for cancer incidence and 8.2 (9.6 million) for mortality rate. ${ }^{1}$ Nearly half of these cases occurred in Asia and are mostly diagnosed at the advanced
Correspondence: Yongshun Gao; Yichao Zheng

Email gaoys@zzu.edu.cn;

yichaozheng@zzu.edu.cn 
stage. ${ }^{2,3}$ Most concerned issue is the 5-year survival rate of GC patients remains at only $5 \%$ to $20 \%$. ${ }^{4}$ The prognosis of $\mathrm{GC}$ is related to its pathological stage, location, tissue type, biological behavior and treatment measures. ${ }^{5}$ Traditional chemotherapy drugs for GC have more side effects, and the technology of targeted therapy is still immature. ${ }^{6}$ Hence, to explore the targeted therapy of GC development is the key to find a new therapeutic strategy.

In recent years, lots of research has reported that histone modification is closely related to the development of GC. ${ }^{7,8}$ Ubiquitination, one of the most important histone modifications has been frequently reported to contribute to the progression of GC. ${ }^{9-11}$ Ubiquitin conjugation is a posttranslational modification where a 76-amino-acid ubiquitin protein is conjugated to a lysine residue on a target protein. $^{12}$ As a reversible process, deubiquitinating enzymes (DUBs) catalyze the release of ubiquitin from ubiquitinated substrates. ${ }^{13}$ Notably, the activity of DUBs directly affects the turnover rate, activity, regeneration and localization of various proteins in cells. ${ }^{14}$ Among these DUBs, ubiquitin-specific protease 8 (USP8) was demonstrated to be involved in breast cancer, ${ }^{15,16}$ lung cancer, ${ }^{17}$ corticotroph adenomas $^{18,19}$ and pituitary adenomas, ${ }^{19,20}$ and these studies suggested that USP8 played an important role in different types of cancer. Nevertheless, no study has reported the impact of USP8 on GC. As a key drug target, epidermal growth factor receptor (EGFR) family of receptor tyrosine kinases consists of four members denoted human epidermal growth factor receptor 1 (HER-1, also known as EGFR), HER-2, human epidermal growth factor receptor 3 (HER-3) and human epidermal growth factor receptor 4 (HER-4), that form homo- and heterodimeric complexes upon ligand binding. ${ }^{21}$ Several studies have shown that EGFR was a substrate of USP8 deubiquitination. $^{22-24}$ USP8 mutations enhance the promoter activity of proopiomelanocortin (Pomc) by stabilizing EGFR signaling. ${ }^{24}$ Pharmacological inhibition of USP8 (DUBs-IN-2) induced degradation of receptor tyrosine kinases including EGFR and HER-2. ${ }^{17}$

It is widely known to all that HER-2 is the most close member related to GC progression. ${ }^{25}$ HER-2 is a protooncogene encodes cell-membrane associated proteins erbb2 receptor tyrosine kinase 2 (ErbB2), which is expressed ubiquitously in all cells and the mutation of this gene is responsible for continuous downstream signaling activation, uncontrolled gene amplification and protein expression in tumor cell, inhibition of programmed cell death, formation of tumor angiogenesis, acceleration of tumor invasion force, weakening the immune system, etc. ${ }^{26,27}$ In this context, as a drug target for $\mathrm{GC}$, the relationship between USP8 and HER-2 is worth exploring.

Here, our data not only identifies co-overexpression of USP8 and HER-2 is closely correlated with poor prognosis in patients, but also provides USP8 inhibitor can promote degradation of HER-2 and inhibit proliferation of HER-2 positive GC cells (NCI-N87) significantly in vitro and metastasis in vivo.

\section{Patients and Methods Clinical Samples and Cell Culture}

Primary GC samples were obtained from 69 patients who were radical resection for $\mathrm{GC}$ at the First Affiliated Hospital of Zhengzhou University, China, from August 1, 2011 to June 30, 2014. Written informed consent from the patients or their relatives, which was approved by the ethics committee of the First Affiliated Hospital of Zhengzhou University, was obtained (No. 2019-KY-93). Each sample consisted of cancer and normal tissue obtained from the same patient. Inclusion criteria: (1) Pathological diagnosis of gastric adenocarcinoma; (2) Pathological stage $\mathrm{T}_{1-4} \mathrm{~N}_{0-3} \mathrm{M}_{0}$; (3) Tissue sections and clinical data were kept intact. Exclusion criteria: (1) Patients receiving preoperative chemoradiotherapy or targeted drug therapy (for example, HER-2 positive received trastuzumab); (2) incomplete clinical data and follow-up of lost patients; (3) During the follow-up period, the patients died of diseases other than GC, such as cardiac and cerebrovascular accidents. Clinical data was followed up by a combination of telephone and medical records. The main endpoint of the study was the survival time of the patients from the date of surgery to the date of death. The last follow-up was on June 30, 2019. The histological classification of gastric cancer was based on 2019 WHO classification of digestive tumors, ${ }^{28}$ and the clinical staging was based on the TNM staging of GC in the 8th edition formulated by the American cancer society (AJCC) and the international union against cancer (UICC) in $2017^{29}$

The human GC cell lines (NCI-N87, MKN-45, AGS, BGC-823, HGC-27 and MGC-803) were purchased from the Shanghai Institutes for Biological Sciences, Chinese Academy of Sciences. And all cells had STR cell identification. All cells were cultured in RPMI-1640 (BI, USA) containing $10 \%$ fetal bovine serum (BI, USA) and incubated in a humidified chamber at $37^{\circ} \mathrm{C}$ under $5 \% \mathrm{CO}_{2}$. 


\section{Antibodies and Reagents}

Anti-USP8 (ab228572) was purchased from Abcam (USA). Anti-HER-2 (4290S), anti-ubiquitin (3936S), anti-PI3K (13666S), anti-p-PI3K (17366S) and anti-ubiquitin (3936S) were obtained from CST (USA). Anti-AKT (BS90044) and anti-p-AKT (BS94006) were purchased from Bioworld (USA). Anti-Ki-67 (AF-0198) was collected from Affinity (China). Rabbit anti-GAPDH (AB-P-R-001) was bought from Goodhere (China). USP8 inhibitor (MB7295), MTT (MA0198) and MG132 (MB4176) were obtained from Meilune (China). Cycloheximide (CHX, 239,763 ) was obtained from Sigma-Aldrich (USA).

\section{RNA Extraction and qRT-PCR Analysis}

Total RNA was extracted from cells with TRIzol reagent, and then cDNA was synthesized using the PrimeScript RT Master Mix kit (QIAGEN, Germany). Quantitative reverse transcription-polymerase chain reaction using ChamQTM Universal SYBR ${ }^{\circledR}$ qPCR Master Mix (Vazyme, China). The PCRs were then performed using the 7500 RealTime PCR System (Applied Biosystems, USA) with the primers as follows: HER-2 forward, 5'-AGCCGCGAG CACCCAAGT-3', reverse, 5'-TTGGTGGGCAGGTAGG TGAGTT-3'. GAPDH: forward 5'-GATGACTACCGTC CACTCC- $3^{\prime}$ and reverse $5^{\prime}$-ACTCTGAAAGCCATACCG $-3^{\prime}$. NCI-N87 cells were used as a mock control $3.5 \times 10^{5}$ cells in 12-well plates (NEST, China) until approximately $90 \%$ confluent.

\section{RNA Interference}

All gastric cancer cells were cultivated in $100 \mathrm{~mm}$ dishes and transfected with USP8-specific siRNA oligonucleotide. The negative control was 5 '-UUCUCCAGAACGUGUCACGUT T-3', the si-USP8 was 5'-CCACAGATTGATCGTACTA AATT-3' (GenePharma). Then the cells were transfected by using Lipofectamine 4000 (Invitrogen) according to the manufacturer's instructions.

\section{Western Blot Analysis}

After cell culture meets the requirements of each experiment, total protein was extracted with radioimmunoprecipitation assay (RIPA) lysis buffer. Gel electrophoresis was then performed, the blot was then incubated with 5\% milk for 2 hours and with primary antibody at $4{ }^{\circ} \mathrm{C}$ for overnight. The second antibody was then applied for 2 hours at room temperature and then the membrane was imaged using enhanced chemiluminescence reagent (Thermo, USA) and X-ray film (Carestream, China).

\section{Cell Viability Assay}

Cell viability was determined using the MTT Cell Proliferation and Cytotoxicity Assay Kit (MTT). MTT could be reduced by some dehydrogenase enzymes in the mitochondria to produce formazan, a dark purple crystalline product that can be completely dissolved in the presence of a specific solvent. For that, initially, $4 \times 10^{3}$ cells were plated in 96-well plates (NEST, China) in $100 \mu \mathrm{L}$ volume of media, after incubating in the incubator for 72 hours, added $20 \mu \mathrm{L}$ MTT per well to a final concentration of $5 \mu \mathrm{g} / \mathrm{mL}$ and incubated at $37^{\circ} \mathrm{C}$ for 4 hours. Then, $150 \mu \mathrm{L}$ DMSO was added to each well and placed on a shaker for $10 \mathrm{~min}$. The absorbance was measured using a microplate reader at a wavelength of $570 \mathrm{~nm}$.

\section{Colony Formation and Transwell Assays} Human GC MGC-803 (600 cells/well) and NCI-N87 (1000 cells/well) cells were seeded into 6-well plates for approximately 10 days in RPMI-1640 medium. Then, the cells were cleaned with PBS and fixed with $10 \%$ formalin and stained with a $0.1 \%$ crystal violet solution. After these, the plates were dried and photographed. The clones with more than 50 cells were numbered and analyzed.

Transwell assays were performed to analyze the GC cell migration and invasion ability. $1 \times 10^{5} \mathrm{GC}$ cell suspension was prepared in $300 \mu \mathrm{L}$ serum-free RPMI-1640 medium and then were seeded onto the upper chambers. $600 \mu \mathrm{L}$ of medium containing $20 \%$ fetal bovine serum (FBS) was placed in the lower chambers. Then, the cells were collected after $36 \mathrm{~h}$. The filters were fixed with $10 \%$ formalin for 30 minutes and stained with a 0.1\% DAPI (MCE, China) solution for 20 minutes at room temperature. The plate was then photographed and analyzed by a highcontent screening system (Thermo, USA).

\section{Immunofluorescence Microscopy}

Human GC MGC-803 (1000 cells/well) and NCI-N87 (1500 cells/well) cells were seeded into 24-well plates with glass slide for $24 \mathrm{~h}$ in RPMI-1640 medium. Then, the cells were cleaned with PBS and fixed with $4 \%$ formalin and permeabilzed with a $0.1 \%$ Triton X-100 solution (Sigma-Aldrich, Germany). The corresponding primary and secondary antibodies were given, and DAPI staining was then performed against light for 30 minutes. At last, 
we observed fluorescence using a confocal microscope (Nikon, Japan).

\section{Immunohistochemical Analysis and Evaluation}

Tissues were subjected to immunohistochemistry (IHC) using a specific antibody for USP8 (1:1000), HER-2 $(1: 800)$. Staining was then performed using the diaminobenzidine (DAB kit) (Zsbio, China, Beijing). Negative control experiments were routinely performed without incubated with specific antibody for USP8 and HER-2. The slices were placed into Aperio AT2 (Leica, USA) for scanning. Aperio image analysis (Leica, Germany) software was used to analyze and establish the scoring template. USP8 was located in the cytoplasm and the cytoplasmic template score was used. HER-2 was mainly located in the cell membrane and was scored by the cell membrane template. Cutoff line: After ranking the scores from high to low, the first 50\% (34 cases) of USP8 was high expression, while the remaining 35 cases were low expression and the first $20 \%$ ( 14 cases) of HER-2 was high expression, while the remaining 55 cases were low expression.

\section{Animal and Xenograft Model}

BALB/c-nu mice (4 weeks old, male) were purchased from Hunan SJA Laboratory Animal Co., Ltd. All animals had adapted for one week before the study and had free access to food and water. NCI-N87 $\left(1 \times 10^{7}\right.$ cells in $100 \mu \mathrm{L}$ PBS) and MGC- 803 cells $\left(5 \times 10^{6}\right.$ cells in $100 \mu \mathrm{L}$ PBS $)$ were injected into the hind flank of the nude mice ( 3 mice per group). The USP8 inhibitor at $2 \mathrm{mg} / \mathrm{kg}$ was administered intraperitoneally 5 days a week. Tumor volume and mice weight were measured every 2 days, and tumor weight was measured after excision on the final day of the experiment. After all, mice were sacrificed, partial tumor tissues were fixed in formalin, embedded in paraffin, and analyzed by immunohistochemistry.

\section{Statistical Analysis}

All the collected data were statistically analyzed using SPSS 22.0 software (IBM, New York, Armonk, USA). The results obtained from cell line experiments and animal assays were analyzed using Student's $t$-test (for two groups) or ANOVA (for more than two groups). The paired $t$-test was used to compare the scores of tumor tissues and normal tissues. Chi-square test was used to analyze the association of the expression of USP8 and HER-2 with clinicopathologic features. The KaplanMeier method was used the survival analyses. Data are presented as the mean $\pm \mathrm{SD} . P<0.05$ was considered significant.

\section{Results}

\section{Co-Overexpression of USP8 and HER-2} is Associated with Poor Prognosis in GC Patients

To determine whether USP8 and HER-2 were associated with gastric cancer, we evaluated the expression pattern of them in cancerous tissue and paracancer tissue of GC from the GEPIA database (http://gepia.cancer-pku.cn),${ }^{30}$ and the result showed that the expression of USP8 and HER-2 in cancerous tissue was higher than that in paracancer tissue (Figure 1A and B). To further clarify the result obtained from the database about the relation of USP8 and HER-2 to the survival and prognosis of GC patients. The analysis of USP8 and HER-2 was performed in paraffin sections of 69 patients. The score values of USP8 and HER-2 showed that the expression of them in cancer tissues was higher than that of adjacent tissues (Figure 1C and D). The result also indicated that USP8 was mainly located in the cytoplasm of gastric cancer cells (Figure 1E) and HER-2 was mainly located on the cell membrane (Figure 1E). Besides, overexpression of USP8 in GC tissues was correlated with vascular tumor emboli, $\mathrm{N}$ stage and pTNM stage. However, the expression of HER-2 was not correlated with clinicopathology (Table 1). Besides, the overall survival rate of GC patients with USP8 overexpression was significantly poorer than that of patients with low USP8 expression (Figure 1F). But there is no statistical difference for the survival time of GC patients with overexpression or low expression of HER2 (Figure 1G). Interestingly, patients with low expression of both USP8 and HER-2 performed better prognosis, while those with overexpression of USP8 and HER-2 performed poor prognosis (Figure 1H). Furthermore, USP8 was positively correlated with HER-2 (Figure 1I). Collectively, these results suggest that USP8 and HER-2 are involved in GC development and progression, and there may be inter-regulation between them.

\section{USP8 Inhibitor Inhibits Proliferation and Metastasis of GC Cell Line NCl-N87}

To investigate the role of USP8 and HER-2 in GC progression, their expressions were evaluated in six GC cell 
A

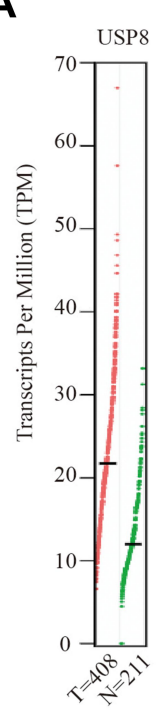

$\mathbf{F}$
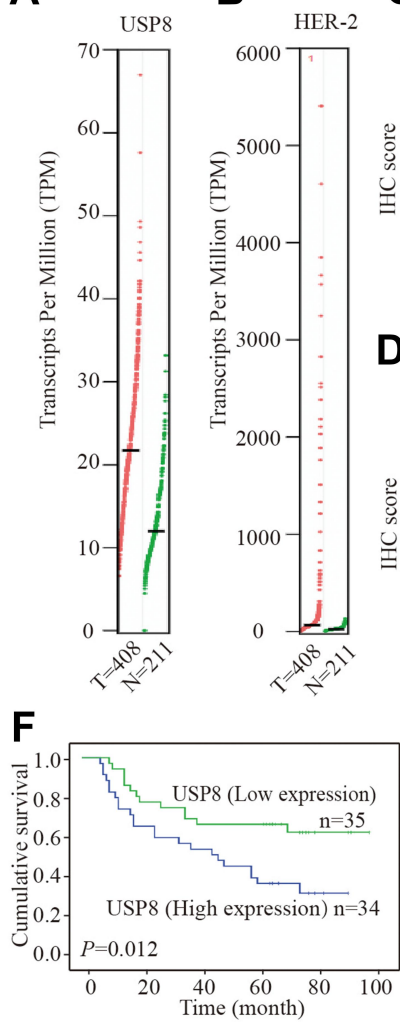

B C

D

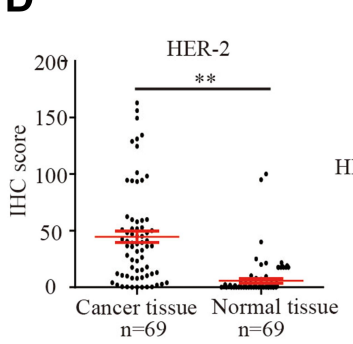

G

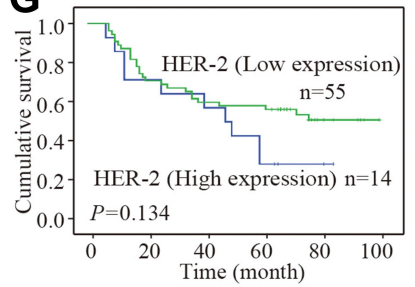

E
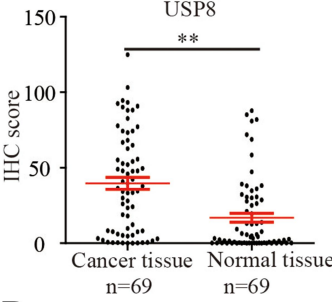

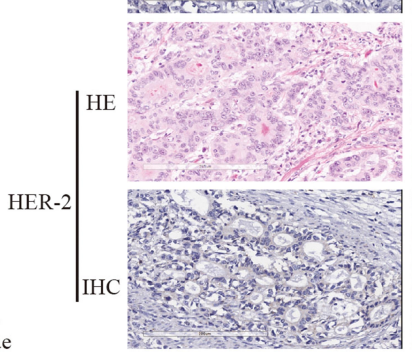

H

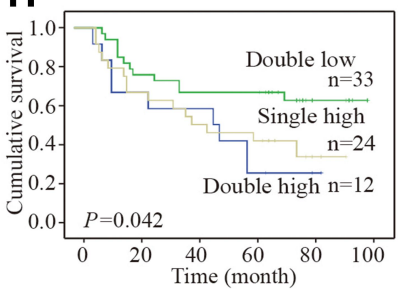

Low expression
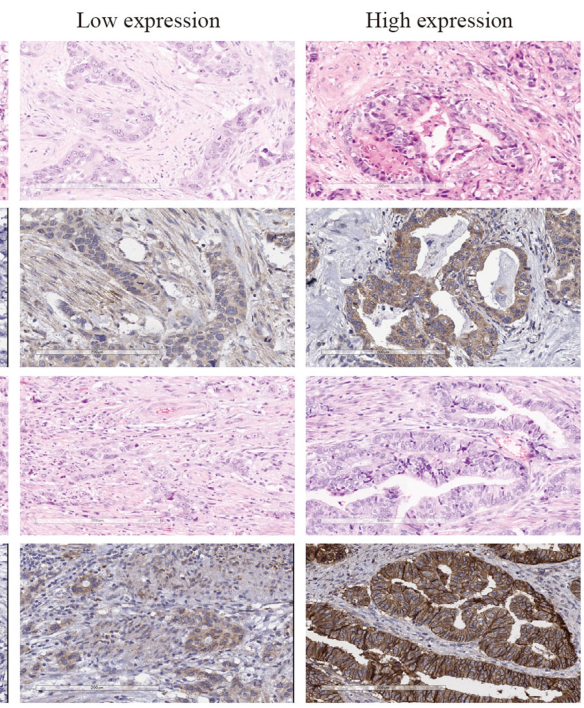

Figure I USP8 and HER-2 co-high expression is associated with poor prognosis in gastric cancer patients. (A) As analyzed by GEPIA, the USP8 expression level in GC tissues was higher than that observed in the corresponding adjacent nontumor tissues. (B) Analyzed by GEPIA, the HER-2 expression level in GC tissues was higher than that observed in the corresponding adjacent nontumor tissues. (C) Analyzed by IHC, the USP8 expression level in GC tissues was significantly higher than that observed in the corresponding adjacent nontumor tissues. ${ }^{* * P}<0.01$. (D) Analyzed by IHC, the HER-2 expression level in GC tissues was significantly higher than that observed in the corresponding adjacent nontumor tissues. **P < 0.0I. (E) The expressions of USP8 and HER-2 in Gastric cancer tissues were evaluated by IHC and HE staining (Hematoxylin-eosin staining, scale bars, 200 $\mu$ m. Kaplan-Meier survival analysis of overall survival in all patients according to USP8 (F), HER-2 (G) and USP8/HER-2 (H) expression. There were three groups in $(\mathbf{H})$, in which USP8 and HER-2 double highly expressed, double low expression and either high expression. The Log rank test was used to calculate $P$ values. (I) Linear regression analysis was used to examine the correlation between USP8 and HER-2 score levels in human GC tissues.

lines, including NCI-N87, MKN-45, AGS, BGC-823, HGC-27 and MGC-803. We found that HER-2 was overexpressed in NCI-N87 only. Therefore, NCI-N87 was selected and MGC-803 was used as a negative control because HER-2 was negatively expressed in this cell line (Figure 2A). In order to find out whether the pharmacological inactivation of USP8 could inhibit the growth of GC cells and was related to HER-2, USP8 inhibitor (Figure 2B) was applied to evaluate the antiproliferation activity of these two cell lines. The result showed that the antiproliferation effect of USP8 inhibitor on NCI-N87 was significantly higher than that of MGC-803 (Figure 2C). Thus, it was confirmed that USP8 inhibitor can inhibit the proliferation of NCI-N87 cell line.

To investigate the anti-cancer activity of USP8 inhibitor in vivo, NCI-N87 and MGC-803 cells were subcutaneously transplanted into nude mice. The mice were then treated with USP8 inhibitor. As shown in Figure 2D and E, USP8 inhibitor significantly suppressed NCI-N87 cell tumor growth, while it failed to inhibit the growth of MGC-803. Similarly, tumor volume in NCI-N87 mice was limited compared to MGC-803 (Figure 2F). Besides, although the USP8 inhibitor prevented tumor growth in NCI-N87 xenografts, the bodyweight of mice bearing MGC-803 and NCI-N87 was not affected significantly (Figure 2G). To further study the effect of USP8 inhibitor on the proliferation of gastric cancer, expression of HER-2 and Ki-67 was examined in MGC-803 and NCI-N87 bearing mice. The results showed that the expression of HER2 and $\mathrm{Ki}-67$ in the USP8 inhibitor group in NCI-N87 was significantly lower than that in the control group, while there was no significant change in the expression of HER-2 and Ki67 in MGC- 803 bearing mice. Specifically, we assume that it is because HER-2 was absent in tumor from mice bearing MGC-803 cells (Figure 2H and I). These results clearly showed that the USP8 inhibitor inhibited the growth of gastric cancer cell NCI-N87 in vivo. 
Table I Expression of USP8 and HER-2 and Patient Characteristics

\begin{tabular}{|c|c|c|c|c|c|c|c|}
\hline \multirow[t]{2}{*}{ Characteristics } & \multirow[t]{2}{*}{ No. } & \multicolumn{2}{|l|}{ USP8 } & \multirow[t]{2}{*}{$P$ value } & \multicolumn{2}{|l|}{ HER-2 } & \multirow[t]{2}{*}{$P$ value } \\
\hline & & High Expression & Low Expression & & High Expression & Low Expression & \\
\hline Gender & & & & 0.960 & & & 0.382 \\
\hline Male & 59 & 29 & 30 & & 13 & 46 & \\
\hline Female & 10 & 5 & 5 & & 1 & 9 & \\
\hline Age (yr) & & & & 0.279 & & & 0.208 \\
\hline$<60$ & 35 & 15 & 20 & & 5 & 30 & \\
\hline$\geq 60$ & 34 & 19 & 15 & & 9 & 25 & \\
\hline Tumor size $(\mathrm{cm})$ & & & & 0.116 & & & 0.813 \\
\hline$<3$ & 18 & 6 & 12 & & 4 & 14 & \\
\hline$\geq 3$ & 51 & 28 & 23 & & 10 & 41 & \\
\hline Location & & & & 0.393 & & & 0.767 \\
\hline Gastroesophageal junction & 37 & 20 & 17 & & 8 & 29 & \\
\hline Others & 32 & 14 & 18 & & 6 & 26 & \\
\hline Differentiation & & & & 0.614 & & & 0.861 \\
\hline Well/Moderate & 16 & 7 & 9 & & 3 & 13 & \\
\hline Poor & 53 & 27 & 26 & & 11 & 42 & \\
\hline Lauren type & & & & 0.555 & & & 0.157 \\
\hline Intestinal & 28 & 15 & 13 & & 8 & 20 & \\
\hline Diffuse & 41 & 19 & 22 & & 6 & 35 & \\
\hline Nerve invasion & & & & 0.118 & & & 0.208 \\
\hline Yes & 30 & 18 & 12 & & 4 & 26 & \\
\hline No & 39 & 16 & 23 & & 10 & 29 & \\
\hline Vascular tumor emboli & & & & 0.041 & & & 0.512 \\
\hline Yes & 30 & 19 & II & & 5 & 25 & \\
\hline No & 39 & 15 & 24 & & 9 & 30 & \\
\hline T stage & & & & 0.387 & & & $0.78 I$ \\
\hline $\mathrm{TI}+\mathrm{T} 2$ & 13 & 5 & 8 & & 3 & 10 & \\
\hline $\mathrm{T} 3+\mathrm{T} 4$ & 56 & 29 & 27 & & 11 & 45 & \\
\hline $\mathrm{N}$ stage & & & & 0.036 & & & 0.199 \\
\hline No & 40 & 24 & 16 & & 6 & 34 & \\
\hline NI-3 & 29 & 10 & 19 & & 8 & 21 & \\
\hline pTNM stage & & & & 0.039 & & & 0.487 \\
\hline I & 10 & 4 & 6 & & 3 & 7 & \\
\hline II & 20 & 4 & 14 & & 5 & 15 & \\
\hline III & 38 & 26 & 15 & & 6 & 33 & \\
\hline
\end{tabular}

Note: $P$ values were calculated by the chi-square test.

Furthermore, we investigated the proliferation and metastasis ability of GC cells in vitro. As shown in Figure 3A, USP8 inhibitor significantly inhibited the proliferation of NCI-N87 cells compared with the MGC. And also, the invasion and migration ability of NCIN87 cells with the inhibitor were significantly reduced according to transwell experiments (Figure 3B). Thus, we believe that USP8 inhibitor suppresses HER-2 positive
GC cells (NCI-N87) proliferation and metastasis in vitro and in vivo.

\section{USP8 Inhibitor Promotes the Degradation of HER-2}

To investigate the effect of USP8 inhibitor on the expression of HER-2, different concentrations of USP8 inhibitor were 
A

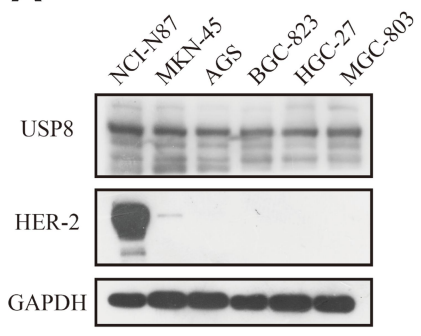

B

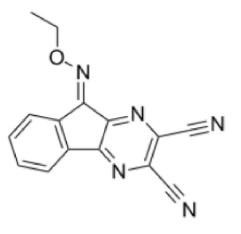

C

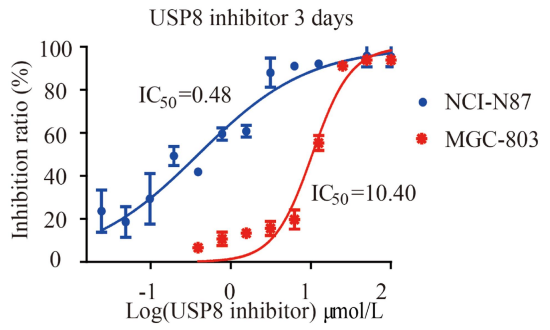

D

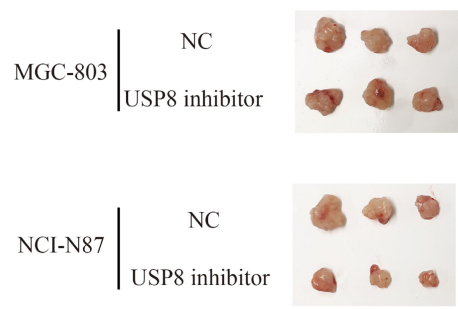

E

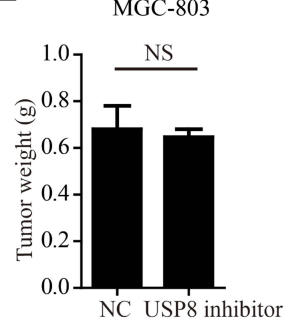

NCI-N87

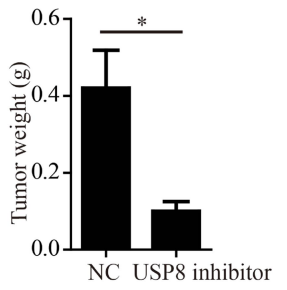

$\mathbf{F}$

MGC-803
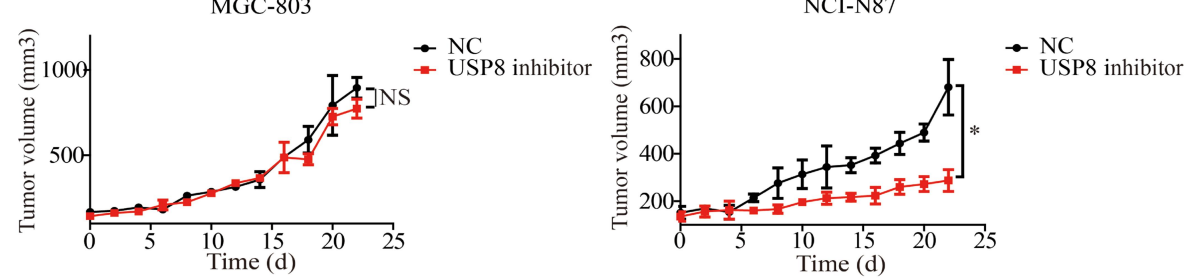

G
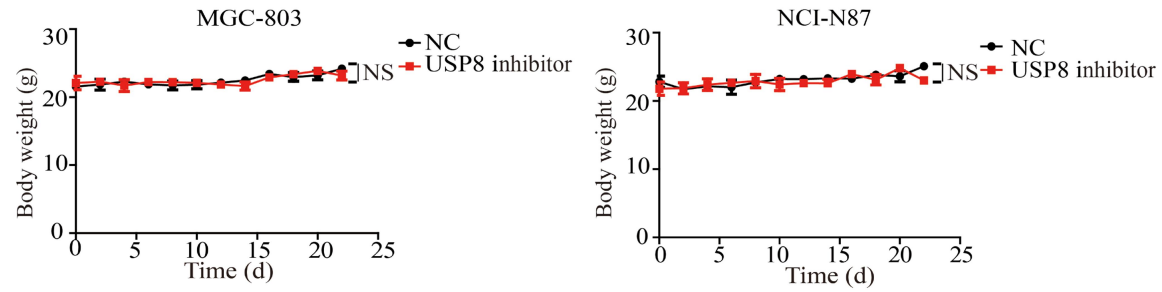

H
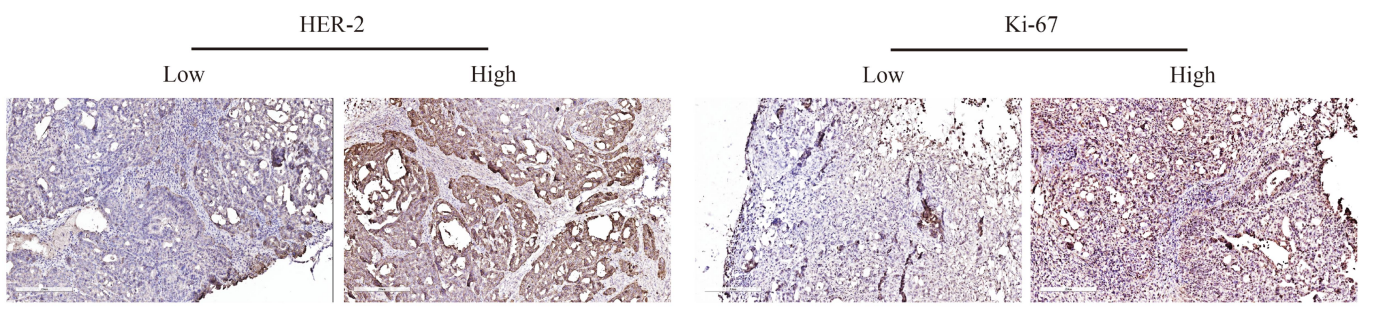

I
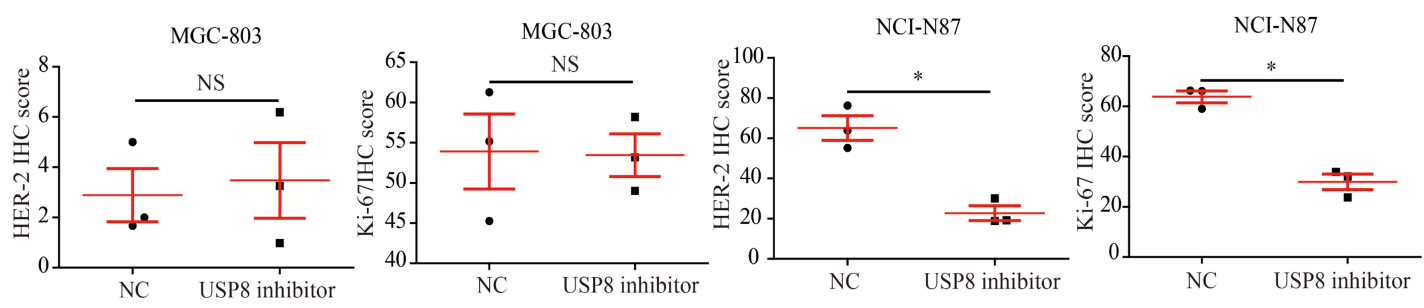

Figure 2 USP8 inhibitor inhibits gastric cancer cell growth. (A) Expression levels of USP8 and HER-2 in six GC lines as indicated. (B) Chemical structure of DUBs-IN-2. (C) The proliferation rates of cells were evaluated with indicated treatment. (D) Representative photographs of NCl-N87 and MGC-803 tumors in nude mice, and $2 \mathrm{mg} / \mathrm{kg}$ USP8 inhibitor was administered intraperitoneally 5 days a week. (E) Bar graph represents the results of the average tumor weight (presented as the mean \pm standard deviation, $\mathrm{n}=3)$. (F) Curve of the tumor volume $\left(\mathrm{mm}^{3}\right)$ of mice. (G) Curve of body weight $(\mathrm{g})$ of mice. ( $\mathbf{H}$ and $\left.\mathbf{I}\right)$ HER-2 and Ki-67 expression levels in tumor from mice bearing MGC803 and NCl-N87 with USP8 inhibitor treatment, and immunohistochemical expression of Ki-67 and HER-2 in MGC-803 and NCI-N87 bearing mice. $* P<0.05$. NS, no statistical significance. 
A
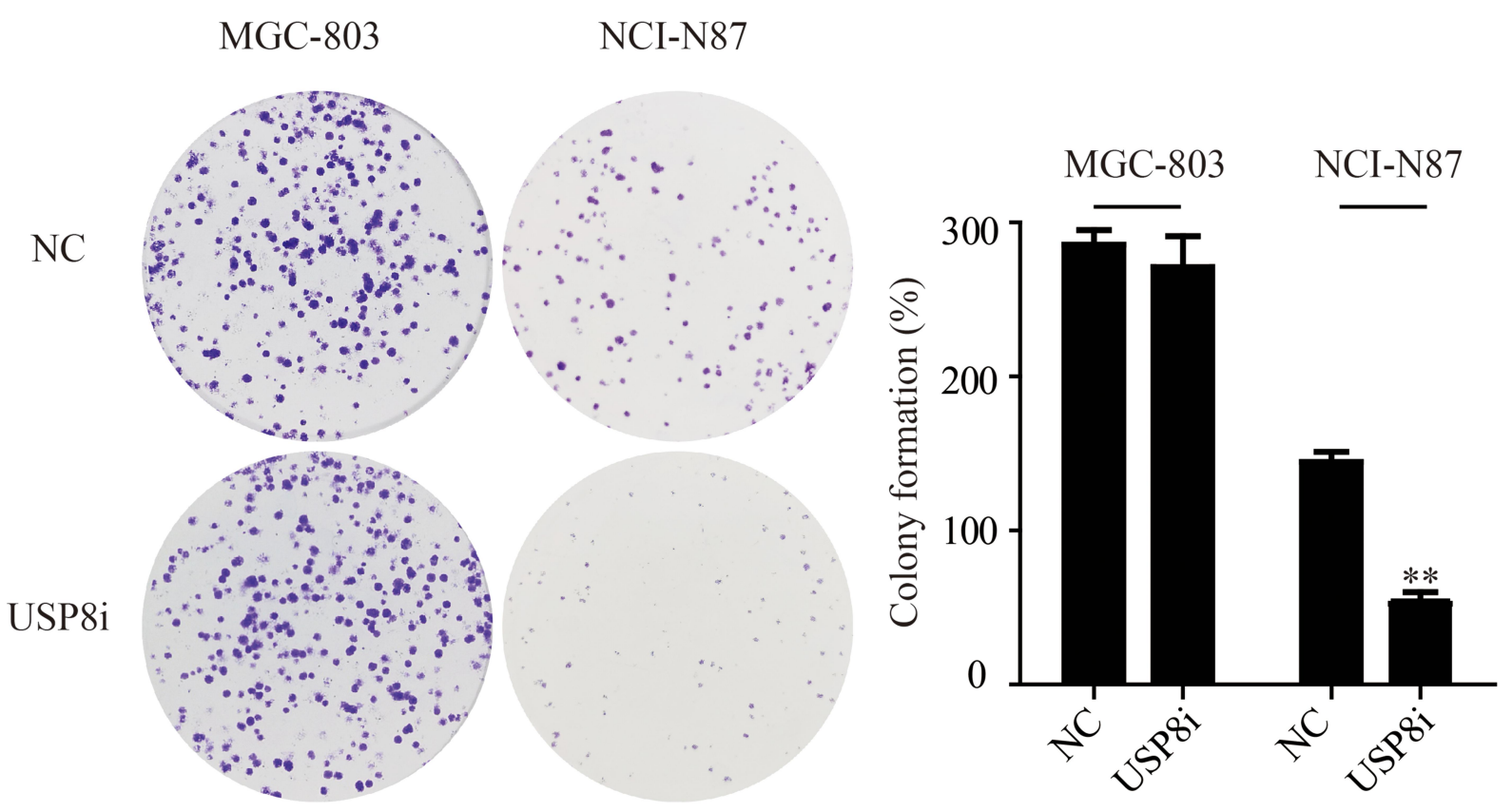

B
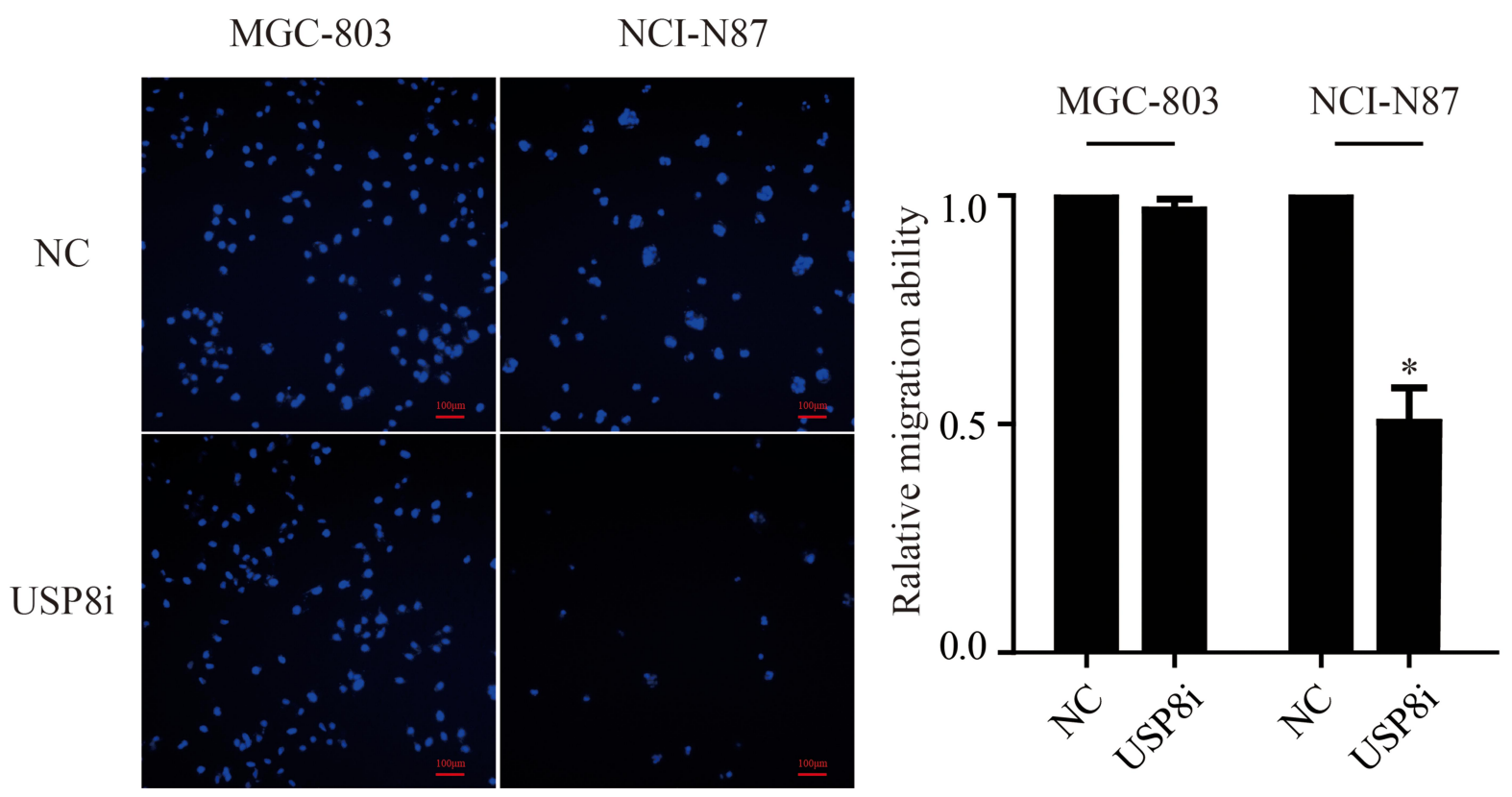

Figure 3 USP8 inhibitor inhibits gastric cancer cell proliferation and metastasis. (A) Colony formation assay of MGC-803 and NCl-N87 cells. Colony numbers were counted and recorded. $* * P<0.01$. (B) Transwell assays of MGC-803 and NCl-N87 cells. $* P<0.05$, scale bars, $100 \mu \mathrm{m}$. USP8i (USP8 inhibitor) groups, treatment of USP8 inhibitor (500 $\mathrm{nmol} / \mathrm{L})$ for 48 hours.

added in NCI-N87 cells. It showed that the expression of HER-2 decreased gradually with the increasing concentration of USP8 inhibitor (Figure 4A). On the other hand, HER2 mRNA level was not affected by USP8 inhibitor (Figure 4B), indicating that the USP8 inhibitor might regulate the stability of HER-2. So, NCI-N87 cells were treated with USP8 inhibitor in the presence of CHX as indicated in Figure $4 \mathrm{C}$ and $\mathrm{D}$, at 6-hour post-treatment, HER-2 protein was almost completely degraded in NCI-N87 cells compared with the control. Immunofluorescence assay showed that USP8 and HER-2 were co-localized at NCI$\mathrm{N} 87$; however, no fluorescence of HER2 was found in 
A

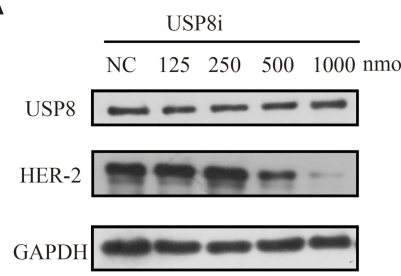

C

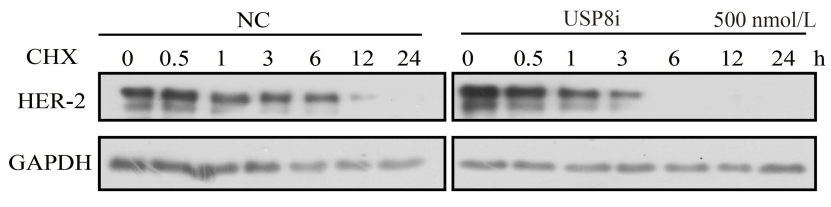

D

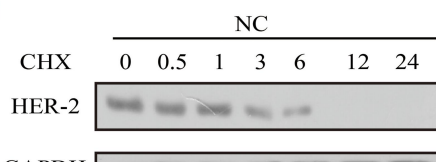

E

DAPI

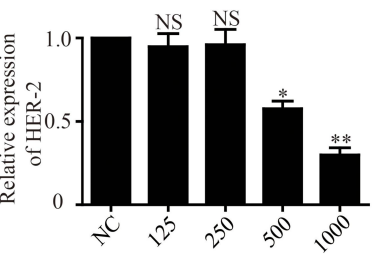

B
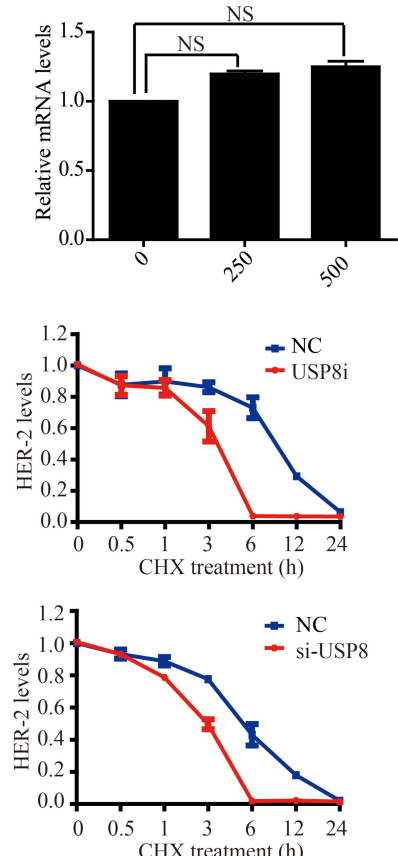

CHX treatment (h)

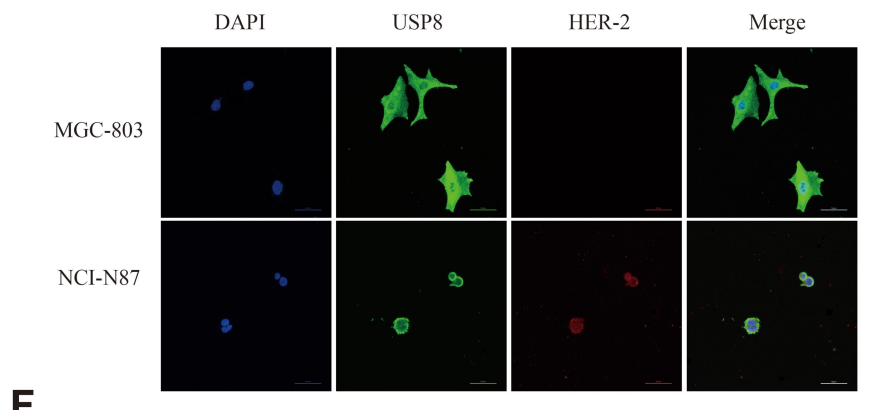

F
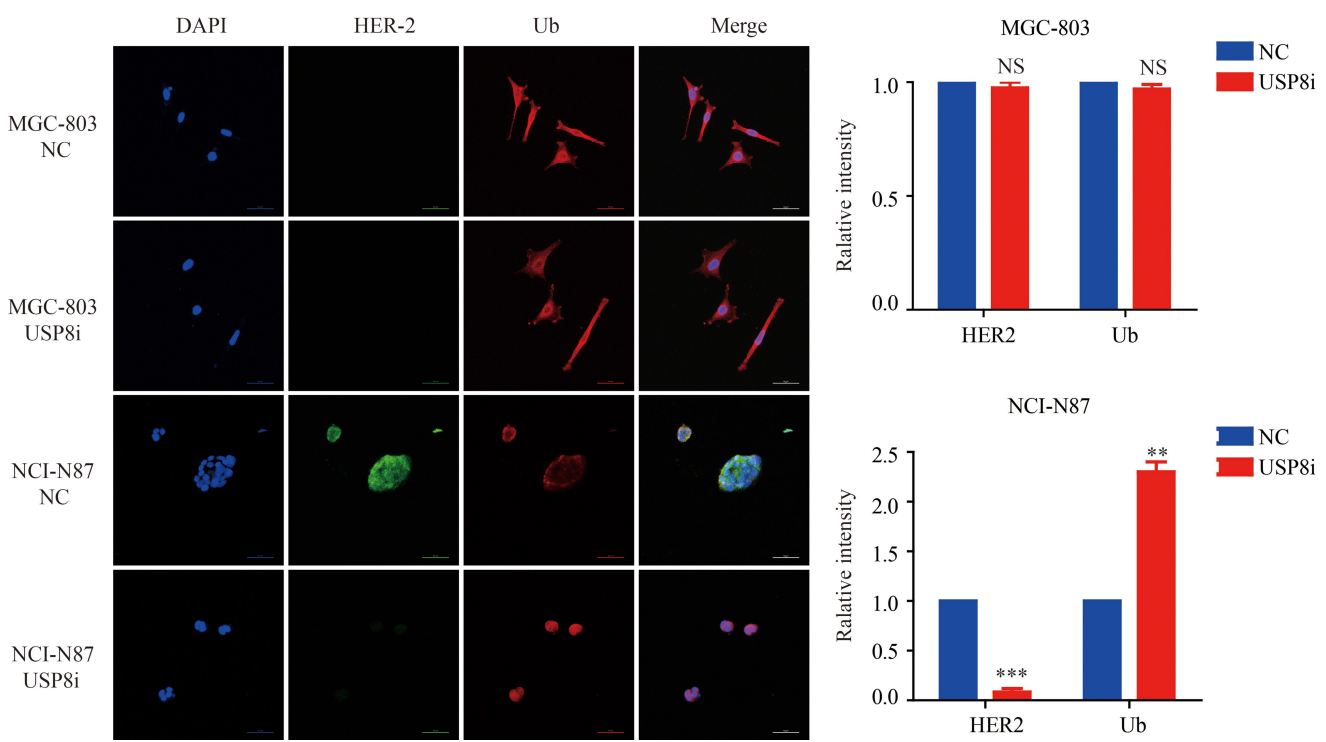

Figure 4 USP8 inhibitor promotes the degradation of HER-2 (A) Expression of HER-2 in the presence of USP8 inhibitor as indicated. (B) mRNA level of HER-2 in NCI-N87 cell line with USP8 inhibitor treatment. Expression of HER-2 in NCI-N87 cells with the treatment of USP8 inhibitor (C) and si-USP8 (D) and cycloheximide (CHX, $20 \mu g$ / $\mathrm{mL}$ ) in combination or alone. (E) Co-localization of USP8 and HER2 in MGC-803 and NCI-N87 cells, scale bars, 50 $\mu \mathrm{m}$. (F) Immunofluorescence of HER-2 and ubiquitin (Ub) changes with treatment of USP8 inhibitor $(500 \mathrm{nmol} / \mathrm{L})$ when compared to negative control (NC), scale bars, $50 \mu \mathrm{m}$. $* P<0.05, * * P<0.01, * * * P<0.001$.

Abbreviation: NS, no statistical significance. 
MGC-803 (Figure 4E). Furthermore, fluorescence intensity of ubiquitin (Ub) was significantly increased and HER-2 reduced with the treatment of USP8 inhibitor, while the MGC803 was not changed at all (Figure 4F). In a nutshell, these data support the notion that USP8 stabilizes HER-2, preventing it from ubiquitin proteasome-mediated degradation.

\section{USP8 Inhibitor Was Involved in PI3K/AKT Signaling Pathway in GC Cells}

We measured the regulatory pathways related to tumor metastasis and growth to clarify the molecular mechanisms of USP8 inhibitor regulating metastasis and growth of GC cell. NCI-N87 with USP8 inhibitor increased the expression of E-cadherin and decreased the expression of N-cadherin, which plays important role in cell metastasis (Figure 5A).

In previous studies, the PI3K/AKT pathway was reported as a major signaling pathway associated with tumor progression and invasion. ${ }^{31,32}$ Therefore, we then examined the expression of PI3K/AKT-signaling-related molecules. The expression of $\mathrm{p}-\mathrm{AKT}$, and p-PI3K was lower in NCI-N87 with USP8 inhibitor group in Figure 5B. All these results demonstrated that USP8 inhibitor was involved in PI3K/ AKT pathway in GC cells.

\section{Discussion}

The important role of DUBs in cell proliferation and metastasis manifests an association between these enzymes and tumorigenesis. Accumulated data has indicated that DUBs are significantly maladjusted in many types of cancers. ${ }^{33-36}$ Until now, role of USP8 in many human cancers is still unknown, and this present study is the first to investigate USP8 in gastric cancer. This study showed that USP8 was overexpressed in both clinical GC tissues and cells, and USP8 overexpression was associated with certain clinicalpathologic variables, such as vascular tumor emboli, $\mathrm{N}$ classification and pTNM stage. All of these clinical studies suggested an association between USP8 and GC. Besides, USP8 was identified to be positively associated with HER-2. Kaplan-Meier analysis demonstrated that GC patients with USP8 and HER-2 overexpression in GC performed a significantly shorter survival time compared to the patients with low expression. Those results confirmed that both USP8 and HER-2 might contribute to the growth of GC.
A

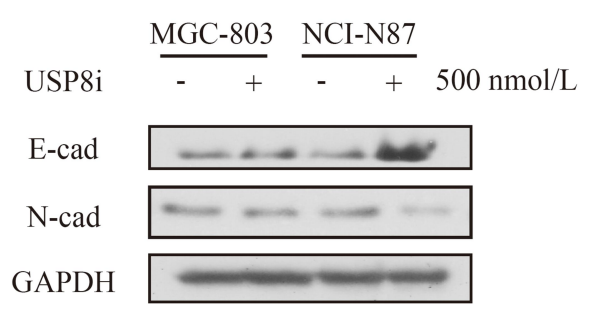

B

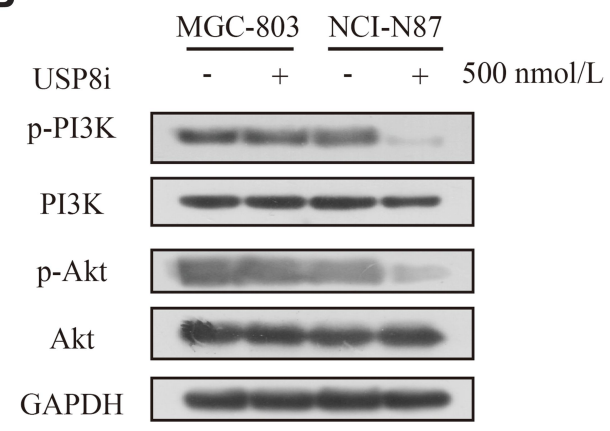

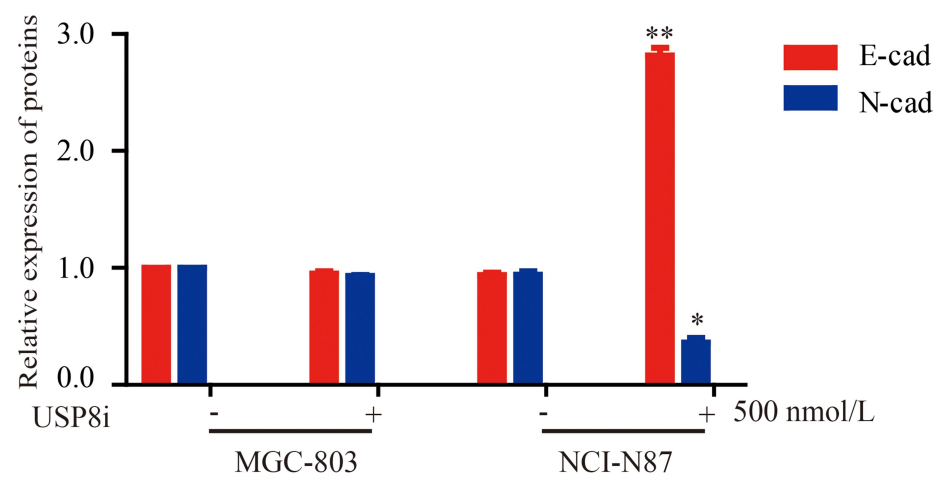

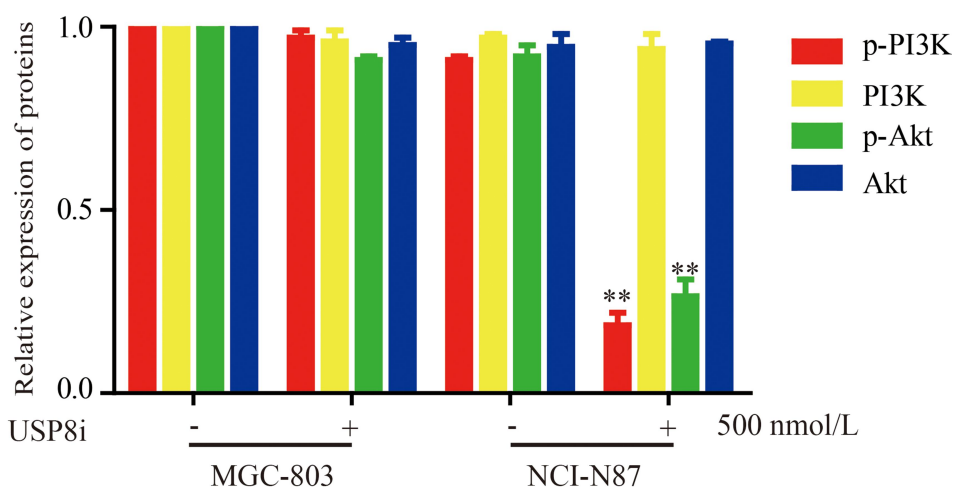

Figure 5 USP8 inhibitor regulates PI3K/AKT signaling in gastric cancer. (A) Protein expression of metastasis-related molecules according to Western blot analysis. (B) Western blotting analysis of PI3K/AKT signaling-related proteins in MGC-803 and NCI-N87 cells. USP8i, USP8 inhibitor. $* P<0.05, * * P<0.01$. 
Moreover, when the USP8 inhibitor was applied to HER-2 positive GC cells and HER-2 negative GC cells which are NCI-N87 and MGC cells, respectively, the growth of NCI-N87 was significantly inhibited. To find out how USP8 regulated the expression of HER-2, thereby regulating the proliferation of GC, NCI-N87 cells were treated with different concentrations of USP8 inhibitor. Results indicated that HER-2 expression was gradually inhibited after treatment with USP8 inhibitor. It was also observed that USP8 inhibitor could inhibit the activation of PI3K/AKT, indicating that USP8 could regulate the HER-2- PI3K/AKT signaling pathway. Furthermore, it was found that USP8 inhibitor did not regulate the transcription level of HER-2, but may promote the ubiquitination of HER-2, resulting in its degradation. Combined with in vitro animal experiments, it was demonstrated that USP8 inhibitor significantly inhibited the growth of NCIN87 cells in vivo but not MGC-803 cells.

Taken together, our findings show for the first time that USP8 inhibitor can be used as a degrader of HER-2 in HER-2 positive GC cells. Moreover, the application of USP8 inhibitor can inhibit HER-2 positive GC cell proliferation and metastasis in vitro and in vivo. In addition, we revealed that the USP8 inhibited GC cell proliferation and migration via the PI3K/AKT signaling pathway, giving a novel insight about USP8 targeting therapy as an alternative approach against HER-2 positive GC cells.

\section{Conclusion}

The USP8 inhibited HER-2 positive GC cell proliferation and migration in vivo and in vitro and probably served as a novel potential therapeutic biomarker for HER-2 positive GC.

\section{Ethical Approval}

All animal experiments as described above were approved by the Institutional Animal Care and Use Committee of Zhengzhou University and performed based on the guidelines of the Institutional Animal Care and Use Committee of Zhengzhou University. The study with human samples was approved by the Ethics Committee of the First Affiliated Hospital of Zhengzhou University. And we clarify that all clinical samples described here were gained from patients who had given written informed consent.

\section{Funding}

This work was supported by National Natural Science Foundation of China (No.81602961, No. 81430085 and No.21372206).

\section{Disclosure}

We declare that we have no conflicts of interest in this work.

\section{References}

1. Bray F, Ferlay J, Soerjomataram I, Siegel RL, Torre LA, Jemal A. Global cancer statistics 2018: GLOBOCAN estimates of incidence and mortality worldwide for 36 cancers in 185 countries. $C A$ Cancer J Clin. 2018;68(6):394-424. doi:10.3322/caac.21492

2. Torre LA, Bray F, Siegel RL, Ferlay J, Lortet-Tieulent J, Jemal A. Global cancer statistics, 2012. CA Cancer J Clin. 2015;65(2):87-108. doi: $10.3322 /$ caac. 21262

3. Etemadi A, Safiri S, Sepanlou SG, Collaborators GBDSC. The global, regional, and national burden of stomach cancer in 195 countries, 1990-2017: a systematic analysis for the Global Burden of Disease study 2017. Lancet Gastroenterol Hepatol. 2020;5(1):42-54. doi:10.1016/S2468-1253(19)30328-0

4. Siegel R, Ma J, Zou Z, Jemal A. Cancer statistics, 2014. CA Cancer J Clin. 2014;64(1):9-29. doi:10.3322/caac.21208

5. Chen Y, Pang X, Ji L, Sun Y, Ji Y. Reduced expression of deubiquitinase USP33 is associated with tumor progression and poor prognosis of gastric adenocarcinoma. Med Sci Monit. 2018;24:3496-3505. doi:10.12659/MSM.908075

6. Russi S, Verma HK, Laurino S, et al. Adapting and surviving: intra and extra-cellular remodeling in drug-resistant gastric cancer cells. Int J Mol Sci. 2019;20(15):15. doi:10.3390/ijms20153736

7. Onder Ö, Sidoli S, Carroll M, Garcia BA. Progress in epigenetic histone modification analysis by mass spectrometry for clinical investigations. Expert Rev Proteomics. 2015;12(5):499-517. doi:10.1586/14789450.2015.1084231

8. Zentner GE, Henikoff S. High-resolution digital profiling of the epigenome. Nat Rev Genet. 2014;15(12):814-827. doi:10.1038/ $\operatorname{nrg} 3798$

9. Han F, Li CF, Cai Z, et al. The critical role of AMPK in driving Akt activation under stress, tumorigenesis and drug resistance. Nat Commun. 2018;9(1):4728. doi:10.1038/s41467-018-07188-9

10. Huang LY, Zhao J, Chen H, et al. SCF(FBW7)-mediated degradation of Brg1 suppresses gastric cancer metastasis. Nat Commun. 2018;9 (1):3569. doi:10.1038/s41467-018-06038-y

11. Shao G, Wang R, Sun A, et al. The E3 ubiquitin ligase NEDD4 mediates cell migration signaling of EGFR in lung cancer cells. Mol Cancer. 2018;17(1):24. doi:10.1186/s12943-018-0784-2

12. Faesen AC, Luna-Vargas MP, Sixma TK. The role of UBL domains in ubiquitin-specific proteases. Biochem Soc Trans. 2012;40 (3):539-545. doi:10.1042/BST20120004

13. Wilkinson KD. Regulation of ubiquitin-dependent processes by deubiquitinating enzymes. FASEB J. 1997;11(14):1245-1256. doi:10. 1096/fasebj.11.14.9409543

14. Zhou MJ, Chen FZ, Chen HC. Ubiquitination involved enzymes and cancer. Med Oncol. 2014;31(8):93. doi:10.1007/s12032-014-0093-6

15. Shin S, Kim K, Kim HR, et al. Deubiquitylation and stabilization of Notch1 intracellular domain by ubiquitin-specific protease 8 enhance tumorigenesis in breast cancer. Cell Death Differ. 2019. doi:10.1038/ s41418-019-0419-1

16. Qiu H, Kong J, Cheng Y, Li G. The expression of ubiquitin-specific peptidase 8 and its prognostic role in patients with breast cancer. $J$ Cell Biochem. 2018;119(12):10051-10058. doi:10.1002/jcb.27337

17. Byun S, Lee SY, Lee J, et al. USP8 is a novel target for overcoming gefitinib resistance in lung cancer. Clin Cancer Res. 2013;19 (14):3894-3904. doi:10.1158/1078-0432.CCR-12-3696

18. Jian F, Cao Y, Bian L, Sun Q. USP8: a novel therapeutic target for cushing's disease. Endocrine. 2015;50(2):292-296. doi:10.1007/ s12020-015-0682-y 
19. Sbiera S, Kunz M, Weigand I, Deutschbein T, Dandekar T, Fassnacht M. The new genetic landscape of cushing's disease: deubiquitinases in the spotlight. Cancers (Basel). 2019;11(11):1761. doi:10.3390/cancers11111761

20. Bujko M, Kober P, Boresowicz J, et al. USP8 mutations in corticotroph adenomas determine a distinct gene expression profile irrespective of functional tumour status. Eur J Endocrinol. 2019;181 (6):615-627. doi:10.1530/EJE-19-0194

21. Yarden Y, Sliwkowski MX. Untangling the ErbB signalling network. Nat Rev Mol Cell Biol. 2001;2(2):127-137. doi:10.1038/35052073

22. Kaiser UB. Cushing's disease: towards precision medicine. Cell Res. 2015;25(6):649-650. doi:10.1038/cr.2015.53

23. Ma ZY, Song ZJ, Chen JH, et al. Recurrent gain-of-function USP8 mutations in cushing's disease. Cell Res. 2015;25(3):306-317. doi: $10.1038 / \mathrm{cr} .2015 .20$

24. Kasahara K, Aoki H, Kiyono T, et al. EGF receptor kinase suppresses ciliogenesis through activation of USP8 deubiquitinase. Nat Commun. 2018;9(1):758. doi:10.1038/s41467-018-03117-y

25. Dijksterhuis WPM, Verhoeven RHA, Meijer SL, et al. Increased assessment of HER2 in metastatic gastroesophageal cancer patients: a nationwide population-based cohort study. Gastric Cancer. 2020. doi:10.1007/s10120-020-01039-7

26. Pernas S, Barroso-Sousa R, Tolaney SM. Optimal treatment of early stage HER2-positive breast cancer. Cancer. 2018;124 (23):4455-4466. doi:10.1002/cncr.31657

27. Yang L, Li Y, Bhattacharya A, Zhang Y. A recombinant human protein targeting HER2 overcomes drug resistance in HER2-positive breast cancer. Sci Transl Med. 2019;11(476): eaav1620. doi:10.1126/scitranslmed.aav1620

28. Nagtegaal ID, Odze RD, Klimstra D, et al. The 2019 WHO classification of tumours of the digestive system. Histopathology. 2020;76 (2):182-188. doi:10.1111/his.13975
29. In H, Solsky I, Palis B, Langdon-Embry M, Ajani J, Sano T. Validation of the 8th edition of the AJCC TNM staging system for gastric cancer using the national cancer database. Ann Surg Oncol. 2017;24(12):3683-3691. doi:10.1245/s10434-017-6078-x

30. Tang Z, Li C, Kang B, Gao G, Li C, Zhang Z. GEPIA: a web server for cancer and normal gene expression profiling and interactive analyses. Nucleic Acids Res. 2017;45(W1):W98-W102. doi:10.10 93/nar/gkx247

31. Geng Y, Xu C, Wang Y, Zhang L. Quiescin sulfhydryl oxidase 1 regulates the proliferation, migration and invasion of human glioblastoma cells via PI3K/Akt pathway. Onco Targets Ther. 2020;13:5721-5729. doi:10.2147/OTT.S255941

32. Zhang N, Xing X, Gu F, Zhou G, Liu X, Li B. Ropivacaine inhibits the growth, migration and invasion of gastric cancer through attenuation of WEE1 and PI3K/AKT signaling via miR-520a-3p. Onco Targets Ther. 2020;13:5309-5321. doi:10.2147/OTT.S244550

33. Xia X, Liao Y, Huang C, et al. Deubiquitination and stabilization of estrogen receptor alpha by ubiquitin-specific protease 7 promotes breast tumorigenesis. Cancer Lett. 2019;465:118-128. doi:10.1016/ j.canlet.2019.09.003

34. Chen X, Zhang X, Cai H, et al. Targeting USP9X/SOX2 axis contributes to the anti-osteosarcoma effect of neogambogic acid. Cancer Lett. 2020;469:277-286. doi:10.1016/j.canlet.2019.10.015

35. Sacco JJ, Yau TY, Darling S, et al. The deubiquitylase Ataxin-3 restricts PTEN transcription in lung cancer cells. Oncogene. 2014;33(33):4265-4272. doi:10.1038/onc.2013.512

36. Xia Y, Wang L, Xu Z, et al. Reduced USP33 expression in gastric cancer decreases inhibitory effects of Slit2-Robo1 signalling on cell migration and EMT. Cell Prolif. 2019;52(3):e12606. doi:10.1111/ cpr. 12606

\section{Publish your work in this journal}

OncoTargets and Therapy is an international, peer-reviewed, open access journal focusing on the pathological basis of all cancers, potential targets for therapy and treatment protocols employed to improve the management of cancer patients. The journal also focuses on the impact of management programs and new therapeutic agents and protocols on patient perspectives such as quality of life, adherence and satisfaction. The manuscript management system is completely online and includes a very quick and fair peer-review system, which is all easy to use. Visit http://www.dovepress.com/ testimonials.php to read real quotes from published authors 